\title{
Undesirable Perioperative Anesthesia Outcomes at Two National Referral Hospitals, a cross sectional study in Eritrea
}

Yonatan Mehari Andemeskel ( $\sim$ Yonimer2@gmail.com)

Department of Anesthesia \& Critical care Nursing, School of Nursing, Asmara College of Health Sciences https://orcid.org/0000-0003-1772-1042

Traudl Elsholz Asmara College of Health Sciences

Ghidey Gebreyohannes

Asmara College of Health Sciences

Eyasu H. Tesfamariam

Asmara College of Health Sciences

Research article

Keywords: Leiden preoperative care patient satisfaction questionnaire (LPPSq), Perioperative care, Surgery, Undesirable anesthesia outcomes.

Posted Date: March 24th, 2020

DOI: https://doi.org/10.21203/rs.2.19220/v2

License: (c) (1) This work is licensed under a Creative Commons Attribution 4.0 International License. Read Full License 


\section{Abstract}

Background: Perioperative undesirable anesthesia outcomes are common among patients undergoing surgery. They may affect body systems and lead into more serious intraoperative and postoperative problems. This research is conducted in the Eritrean National Referral Hospitals with the aim of assessing the prevalence of undesirable anesthesia outcomes during the perioperative period. Method: A cross sectional study design was applied on 470 patient who underwent different types of surgeries with in a three month period. Patients were interviewed 24 hours after operation using the Leiden preoperative care patient satisfaction questionnaire (LPPSq). This study reports one component of a large study conducted. The dimension "Discomfort and needs" of the LPPSq was considered and the measurement of that dimension are presented in this report. Items of the dimension were standardized and measured using a five point Likert scale from "Not at all" to "Extremely". Multivariable logistic regression was used to look for the association of the outcomes with the types of surgery and types of anesthesia using SPSS (Version 22). Statistical significance level was set at $p<0.05$. Results: The prevalence of undesirable anesthesia outcomes were computed in two manners; prevalence of those with 'at least a little bit' outcomes which was computed to see the total occurrence of these outcomes and prevalence of those having 'more than moderate' outcomes to see the severe experience of these outcomes. Prevalence of the predominant undesirable outcome, post-operative pain, for 'at least a little bit' and 'more than moderate' were $82.6 \%$ and $43.6 \%$ respectively. The rest post-operative undesirable outcomes were less frequently reported. Conclusion: Postoperative pain was found to be the most prevalent undesirable outcome. Enhancement of the proper assessment and management of postoperative pain through the development and implementation of specific pain management modalities is needed. Local guidelines should also be available in these settings for the treatment of acute postoperative pain or other outcomes. Keywords: Leiden preoperative care patient satisfaction questionnaire (LPPSq), Perioperative care, Surgery, Undesirable anesthesia outcomes.

\section{Background}

Undesirable peri-operative anesthesia symptoms are common and may affect all patient's body systems (1). Among the most commonly mentioned complications are pain, nausea, vomiting, sore throat, shivering, thirst and hunger (1-3). It has been observed in the Eritrean National Referral hospitals that these complications to trouble surgical patients during their perioperative stay. Common problems that have been observed to be associated with their occurrence are underestimation of these undesirable events as well as lack of adequate management protocols and skills. However, failing to prevent these undesirable events have been linked to subsequent, more serious intraoperative and postoperative problems (2). They have also been explained as modifiable sources of patient dissatisfaction (3).

General anesthesia deprives the patient's consciousness and the abolition of sensation and reflexes (1). Despite its increasing safety, it comes with certain risks and complications that have been recognized and dealt with (2). The side effects of local anesthetics occur as a result of suppression of the cellular conduction and these effects occur within five to ten minutes right after the injection is given (1). In case 
they are administered intravenously, the reaction appears immediately. Patients may experience headache, back pain associated with needle insertion, postoperative surgical site pain, pain that radiates from the diseased or injured area, and even the pain may occur during medication administration (1).

Postoperative pain is a common experience for postoperative patients and remains a serious problem facing anesthesia providers in their daily practice (4). Poor management of acute postoperative pain is among the causes for some medical complications. Furthermore, it interferes with postoperative recovery, prolongs the time of hospitalization, and increases postoperative morbidity $(5,6)$. However, analgesia should be the fundamental right of every patient. Furthermore, proper management of postoperative pain is known to significantly reduce morbidity during the perioperative period, including the incidence of postoperative complications, hospital stay and cost $(6,7)$. It has also been emphasized the importance of postoperative pain management during the early stage of recovery $(8,9)$. Not only during the postoperative period but adequate pain management plan requires preoperative preparation; up until after discharge to control pain effectively (10). It has been found out that pre-administered analgesia prevents or reduces the analgesic requirement (11). Moreover, accuracy in pain assessment is a major factor in measuring the adequacy of pain management $(4,12)$.

Postoperative nausea and vomiting (PONV) is another frequently occurring unpleasant experience that often occurs after surgery (13). It has been reported that it is more related or occur with general anesthesia than when using regional anesthesia $(2,14,15)$. Hypothermia is also another undesirable event that occurs frequently affecting more than $70 \%$ of patients undergoing surgery and anesthesia (14). Exposure to cold temperature during the intraoperative period, administration of unwarmed intravenous fluids, and evaporation from within the surgical incision are the main factors that may lead to hypothermia $(2,16)$. The deactivation of thermoregulation center by general anesthesia itself can also lower the core temperature. Moreover the usage of muscle relaxants during the operation greatly affects the patient's muscles ability to shiver and produce heat thus resulting in temperature drop (16). This would be more pronounced in settings with limited resources like the operating rooms in the selected Eritrean study settings that otherwise would help to maintain the temperature of the rooms and of that of the patient. It is mandatory to monitor body temperature of patients undergoing general anesthesia exceeding 30 minutes in duration or longer (16). Yet this is not a routine practice in the selected study settings.

Perioperative outcomes are determined by the quality of care provided during the perioperative period (2). Recognizing and treating these complications is vital in the provision of good quality of care $(1,2)$. The effectiveness of management is determined by the correct and timely detection of the undesirable perioperative anesthesia symptoms and applying appropriate pharmacotherapy. While managing those symptoms considering the pharmacodynamics and pharmacokinetics of the drugs used as well as their possible interactions, complications is very important (1). Moreover, patients should be provided with adequate information during their preoperative evaluation about anaesthesia, pain relief or any other service provided by anaesthetists so that they can make informed decisions about their treatment and 
care (17). The previous study done in the selected settings found out that information provision is low and it underscores the importance of information and communication (18).

A patient who undergoes anesthesia is at risk of complications and the anesthetist is expected to be responsible in securing patient safety through adequate management of these risks and outcomes (19). Moreover, when a patient experiences anaesthetic-related complication in the recovery room it is the duty of the anaesthetist to ensure that the patient is made aware of and fully understands the complications $(20,21)$. Meanwhile, in Eritrea the work of anesthetists is usually confined only to the operating room. No anesthetist is involved in the management of patients in the postoperative recovery rooms unless the patient underwent in to an unexpected emergency condition that require emergency management. Therefore meaningful anesthetic evaluations and management of postoperative patient outcomes is hardly a practice in these settings. Although no research exits regarding the perioperative undesirable experiences and their management, pain is undervalued and insufficient knowledge of pain management persists. Moreover, no local standard guideline exits in the management of postoperative pain or any other postoperative undesirable event. Therefore this study is aimed at determining the prevalence of perioperative anesthesia related undesirable outcomes among patients undergoing surgery in the selected hospital settings.

\section{Methods}

\section{Study design and Setting}

This descriptive, cross sectional study was conducted between January and March of 2018 in Eritrea, a country in the horn of Africa. Eritrea has two National Referral Hospitals which are located at the Capital city, Asmara. They are called Halibet and Orotta National Referral Hospitals and both of them provide health services at a tertiary level. They are the only governmental medical surgical national referral hospitals in which all types of major and minor surgeries take place.

\section{Participants}

During the study period, a total of 526 patients underwent surgeries under general and regional anaesthesia. The eligibility to participate in the study was based on the respondent's willingness to take part in the study. The flow diagram that shows the ultimate eligible participants $(n=470)$ in the study is discussed elsewhere (18).

\section{Data collection tool and method}

The key elements of socio-demographic and clinical characteristics of the patients were obtained using a socio-demographic and clinical form. The socio-demographic and clinical details obtained were age, 
gender, place of residence (urban or rural), occupation, hospital setting, health coverage, type of anesthesia, type of surgery, and admission type (emergency or elective). The undesirable anesthesia outcomes were measured using the dimension "discomfort and needs" of the Leiden Perioperative Patient Satisfaction questionnaire (LPPSq). The LPPSq was initially modified by Calijouw et al. (2008) and permission was asked and obtained from the responsible author. The LPPSq is a validated suitable for research scale (22), having six dimensions, in which 'discomfort and needs' was separately handled and analysed because of its unique psychometric characteristics $(23,24)$. As far as the founders of the scale are concerned, the internal consistency within the discomfort and need dimension was so low that, it was not incorporated to get a composite score of the LPPSq, resulting to analysis of the individual items separately. This of course was one of the clear indications that the items listed in the dimension provide wider objectives and another perspective of anesthesia service. Explanation of the five other dimensions is made in previous publication (18). The 'discomfort and needs' investigates the common adverse outcomes of anesthesia raised from patient's perspective including post-operative pain, sore throat, back pain, nausea, vomiting, cold, hunger, thirst, and headache.

Data was collected through face to face interview by four well trained anaesthetists who do not work in the study settings. They were also assistant researches and were well aware of the study objectives.

\section{Data Collection Procedure}

The researchers visited each hospital and explained the purpose of the study and its clinical significance to the hospital directors after getting the ethical clearance approval by the Research and Human Resources Development, Ministry of Health. Permission to conduct the study was then obtained from each hospital director. Recruitment of the patients was undergone before the moment of their operation. After full explanation of the study objectives and assurance of confidentiality and anonymity, patients were given written informed consent. The interview was then conducted in their respected postoperative wards after making sure that they are free from acute postoperative discomfort. Data was collected through face to face interview and the time to complete the questionnaire was about 15 to 20 minutes. The interview was done with purpose of assessing the status of undesirable perioperative anesthesia outcomes using the questionnaire.

\section{Variables and Measures}

The items in the 'discomfort and need' were standardized and measured using a five point Likert scale. Patients had to state to which degree they experienced each of the attribute stated in each item after operation. The replies to the items were "Not at all" (0), "A little bit" (1), "Moderately" (2), "Quite a bit" (3), and "Extremely" (4). The items were then computed in to two sequences of prevalence; prevalence of those with 'at least a little bit' undesirable anesthesia outcome occurrence which was computed to see the total occurrence of these undesirable outcomes and prevalence of those having 'more than moderate' 
perioperative undesirable anesthesia outcome occurrences to see the severe occurrence of these outcomes.

\section{Validity and Reliability}

Content validity of the items of the dimension was checked along with the rest of the dimensions by expert opinion from the anesthesia department. Translation (to the local language) was done by bilingual language expert and was then back translated to English by another bilingual person who was not aware of the study objectives. A pre-test was also done to ascertain the comprehension and understandability of the questions. Internal consistency of the dimension was also checked (Chronbach $a=0.66$ ).

\section{Data Analysis}

Responses were coded and entered into SPSS (Version 22) statistical software for analysis. Data cleaning and preliminary explorations were done to assure accuracy of entry before conducting the main analysis. Frequency (percentage) and mean (standard deviation) was used to summarize the demographic and clinical variables of the participants. The prevalence of those with 'at least a little bit' outcome occurrence and prevalence of those with 'more than moderate occurrence' were also computed. Moreover, odds ratios $(95 \% \mathrm{Cl})$ were computed to assess the association of undesirable anesthesia outcomes with the types of surgery and types of anesthesia using multivariable logistic regression. $P$ values less than 0.05 were considered as significant throughout the analyses.

\section{Results}

\section{Population Characteristics}

The demographic and clinical details of the participants are shown in Table 1. The age of the respondents ranged from 18 to 85 years, with a mean \pm SD value of $45.9 \pm 14.7$. From the total 470 participants, $55.1 \%$ were males and $44.9 \%$ were females. The majority $(63.2 \%)$ of the patients were from Orotta Hospital. The patients underwent a wide range of surgical procedures, including general, orthopaedic, Gyn/obs, ENT and burn surgery. 267 (56.8\%) patients had general anaesthesia, and 203 (43.2\%) regional anaesthesia.

Table 1. Demographic and clinical Characteristics of the participants 


\begin{tabular}{|c|c|c|c|}
\hline Variables & & Frequency & Percentage \\
\hline \multicolumn{4}{|l|}{ Gender } \\
\hline & Male & 259 & 55.1 \\
\hline & Female & 211 & 44.9 \\
\hline \multicolumn{4}{|l|}{ Residence } \\
\hline & Urban & 274 & 58.3 \\
\hline & Rural & 196 & 41.7 \\
\hline \multicolumn{4}{|c|}{ Occupation } \\
\hline & Employed & 235 & 50 \\
\hline & Unemployed & 235 & 50 \\
\hline \multicolumn{4}{|c|}{ Hospital Setting } \\
\hline & Halibet & 173 & 36.8 \\
\hline & Orotta & 297 & 63.2 \\
\hline \multicolumn{4}{|c|}{ Health coverage } \\
\hline & Paying & 358 & 76.2 \\
\hline & Free & 112 & 23.8 \\
\hline \multicolumn{4}{|c|}{ Type of Anesthesia } \\
\hline & General & 267 & 56.8 \\
\hline & Regional & 203 & 43.2 \\
\hline \multicolumn{4}{|c|}{ Type of Surgery } \\
\hline & General & 261 & 55.5 \\
\hline & Orthopedic & 99 & 21.1 \\
\hline & Gyn/Obs & 89 & 18.9 \\
\hline & ENT & 7 & 1.5 \\
\hline & Burn & 14 & 3.0 \\
\hline \multicolumn{4}{|c|}{ Admission type } \\
\hline & Emergency & 109 & 23.2 \\
\hline & Elective & 361 & 76.8 \\
\hline \multirow[t]{2}{*}{ Age } & Mean & $\mathrm{SD}$ & \\
\hline & 45.87 & 18.53 & \\
\hline
\end{tabular}

\section{Undesirable Anaesthesia Outcomes}

Table 2 shows the prevalence with 'at least a little bit' and 'more than moderate' undesirable anaesthesia outcomes. The predominant undesirable anesthesia outcome was post-operative pain, followed by cold, nausea, vomiting, thirst, back pain, headache, sore throat and hunger. The prevalence of 'at least a little bit' and 'more than moderate' post-operative pain were $82.6 \%$ and $43.6 \%$ respectively.

Table 2. Prevalence of undesirable anaesthesia outcomes $(n=470)$ 


\begin{tabular}{|c|c|c|}
\hline \multirow[b]{2}{*}{ Discomfort \& needs } & \multicolumn{2}{|c|}{ Prevalence of UAO } \\
\hline & $\begin{array}{c}\text { At least a } \\
\text { little bit } \\
\text { n (\%) }\end{array}$ & $\begin{array}{c}\text { More than } \\
\text { moderate } \\
\text { n (\%) }\end{array}$ \\
\hline Post-operative pain & $388(82.6)$ & $205(43.6)$ \\
\hline Sore throat & $87(18.5)$ & $10(2.1)$ \\
\hline Back pain & $125(26.6)$ & $20(4.3)$ \\
\hline Nausea & $191(40.6)$ & $44(9.4)$ \\
\hline Vomiting & $177(37.7)$ & $47(10)$ \\
\hline Cold & $194(41.3)$ & $80(17)$ \\
\hline Hunger & $86(18.3)$ & $21(4.5)$ \\
\hline Thirst & $130(27.7)$ & $47(10)$ \\
\hline Headache & $118(25.1)$ & $25(5.3)$ \\
\hline
\end{tabular}

\section{Association of outcomes with types of surgery and types of anaesthesia}

As shown in table 3, association of the perioperative undesirable outcomes with the types of surgery the patients underwent and the types of anaesthesia those patients took was computed. The odds of nausea $(\mathrm{OR}=1.74,95 \% \mathrm{Cl}: 1.04,2.90)$ and vomiting $(\mathrm{OR}=2.03,95 \% \mathrm{Cl}: 1.19,3.47)$ were higher among patients who took general anesthesia as compared to those who took regional anesthesia. On the other hand, the odds of back pain was $51 \%(\mathrm{OR}=0.49,95 \% \mathrm{Cl}$ : $0.27,0.88)$ and $67 \%(\mathrm{OR}=0.33,95 \% \mathrm{Cl}: 0.14,0.80)$ less among patients who took general anesthesia as compared to those who took regional anesthesia in those who have undergone general surgery and gynecology \& obstetric surgery, respectively.

Table 3. Occurrence of undesirable anaesthesia outcomes (Types of surgery versus types of anaesthesia)

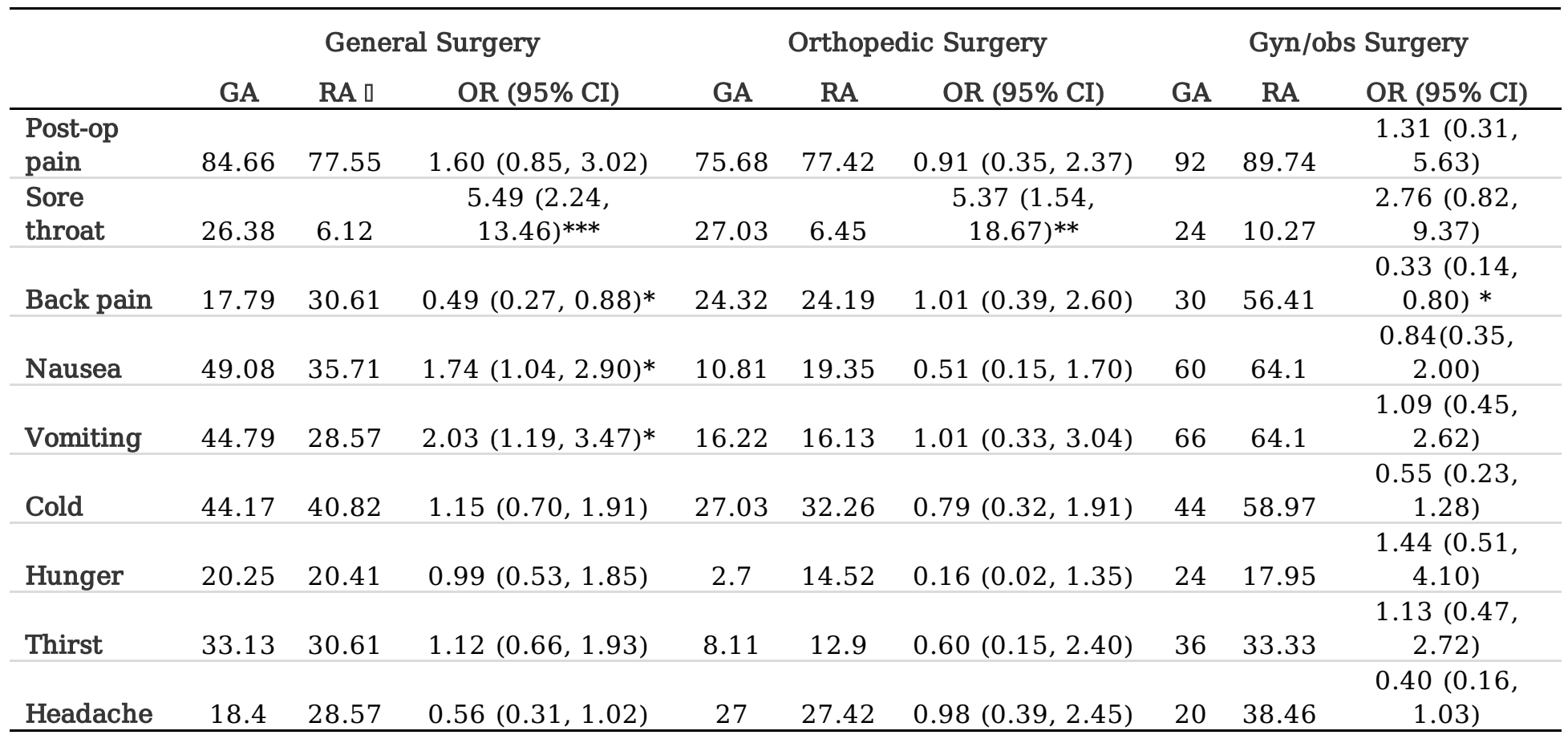

RA(Regional Anesthesia)-Reference category

${ }^{*} p<0.05,{ }^{* *} p<0.01,{ }^{* * *} p<0.001$ 


\section{Discussion}

This is the first survey in Eritrea that discusses about undesirable perioperative anesthesia related events. It focuses on the most common undesirable perioperative anesthesia events raised from patients' perspectives. Generally the management of these events is usually under estimated, especially when it comes to the management of postoperative pain. The involvement of the anesthetist is very important in the management of these events. Moreover, having a local standard guild line is an important strategy in promoting standardization of procedures and patient controlled management. However, these two factors are not yet in practice in the study settings. The work of the anesthetist doesn't go beyond the operating room and the management of these events is left either to the surgeon responsible or to the postoperative nurses. Moreover, limitation in skilled postoperative nurses, shortage of drugs and other necessary equipments are among the commonly mentioned problems.

The provision of adequate pain relief after surgery is an ethical responsibility of healthcare providers and a fundamental right of every patient (25-27). Postoperative pain can have a significant effect on patient recovery by improving clinical outcomes, avoiding clinical complications, and finally saving health care resources (28). Moreover, effective postoperative pain (POP) management is an essential component in the provision of quality of care (29). Nevertheless, it has been explained that appropriate postoperative pain management is generally neglected in Eritrea. Lack of skilled professionals and pain management guild lines also contribute to the condition. In reflection to this, the experience of postoperative pain was found to be high in which the total experience of pain was $82.6 \%$, of whom $43.6 \%$ experienced more than moderate pain. Not only in this study, but also in the studies done using the same questionnaire postoperative pain was among the most frequently experienced complaints $(23,24,30,31)$. Similar results were also reported in another study from the Netherlands by Kalkman and colleagues. This Dutch study was conducted in a university hospital on 1416 patients undergoing various surgical procedures with the aim of developing a validated prediction rule for the occurrence of early postoperative severe pain in surgical patients and the incidence of severe pain was found to be $25.8 \%$, measured one hour after surgery (32). Another similar result was also reported in a study done in Saudi Arabia. The study was conducted on 199 patients who did surgeries in a unuversity hopsital. The same questionnaire (LPPSq) was used and they found out postoperative pain to be the most frequenly mentioned complaint (30). In the studies done by Jlala et al (2010) and Calijouw et al. (2008), which were conducted with the primary aim of validating the LPPS questionnaire, thirst was another frequently mentioned complaint unlike in this current study. The other postoperative side effects (back pain, headache, hunger, nausea and vomiting) were less frequently reported with the lowest median score in all the above mentioned studies including this current study. While according to a study done in Rwanda, which was once again conducted using the same LPPS questionnaire on 145 patients, thirst and hunger were highly reported (31). The status of postoperative pain was also compared between the two types of anesthesia, however no significant difference was scored in its occurrence. Unlike in this study, in a study done by Caljouw et al., 2008, a significant difference was observed in the occurrence of postoperative pain between general anesthsia (82.1\%) and regional anesthesia (34.3\%). 
One of the first unpleasant symptoms in the perioperative period are postoperative nausea and vomiting $(1,13)$. Despite anesthetic and pharmacological advances, there is no drug that is completely effective in preventing post-operative nausea and vomiting (PONV). They appear frequently and they can often be complex and can be a significant problem in anesthesia practice. It is also often described as to be the second most common complaint after pain during the postoperative period $(13,33)$. Understanding the mechanism and careful assessment of risk factors help in its management $(2,33,34)$. Normally, the occurrence of these symptoms is expected to be more commonly seen in those patients who took general anaesthesia while back pain in those who took spinal anaesthesia which is associated with the procedure. Despite these facts, in this study, the occurrence of nausea and vomiting was found to be higher in those patients who took spinal anaesthesia. Meanwhile, as general anaesthesia involves manipulation of the airway, it is obvious that the occurrence of sore throat to be higher in those patients who took general anesthesia. The very few sore throat that occurred among those who took regional anaesthesia may not be purely associated with the anaesthetic procedure. Moreover, the type of anaesthesia given to all those who took regional anaesthesia was spinal and thus the experience of back pain was high among these groups. There wasn't any significant difference in the occurrence of the rest of undesirable anaesthesia outcomes like cold, hunger, thirst and headache.

Generally, perioperative undesirable events still persist despite the medical advances. These events are more prevalent in settings with limited resources. Pre-operative assessment is an important component of anaesthesia (35). Preparing a patient for anaesthesia requires an understanding of the patient's preoperative status, the nature of the surgery and the anaesthetic techniques required for surgery, as well as the risks that a particular patient may face during this time (36). Problems with adequacy of preoperative assessment in the study settings which was mentioned in a previous publication from this study may also contribute to the increment in the prevalence of these undesirable outcomes.

\section{Limitations of the study}

Some of the exclusion criteria may have impacted the results of the study and the fact that the participants were made to be interviewed before their discharge, the dependence of care might have retrained them from speaking their mind.

\section{Conclusion}

Postoperative pain was the most predominant undesirable anesthesia outcome. This gives a signal for the study settings to consider the involvement of anesthetists or dedicated pain nurses in the management of postoperative pain. This would enhance the proper management of postoperative pain through the development and implementation of specific pain management modalities. Local guidelines should be available in these settings for the treatment of acute postoperative pain. Moreover, measuring anesthetic outcomes in a reliable and valid manner would also be important for improving the standards of anesthesia practice and delivering the quality of anesthsia in these settings. It has also been reported 
that adequate information is not provided to patients during the perioperative period regarding their perioperative experience. Therefore, it is required for the study settings to make efforts on providing consistent preoperative information regarding choices for anaesthesia, the risks and benefits of the drugs, post-operative analgesia, prevention, treatment of other outcomes and obtaining maximum efficacy from the administered treatments.

\section{List Of Abbreviations}

ENT: Ear, Nose and Throat; Gyn/Obs: Gynecology and Obstetrics; LPPSq: Leiden Perioperative Care Patient Satisfaction questionnaire. PONV; Postoperative nausea and vomiting; UAO: Undesirable Anesthesia Outcomes.

\section{Declarations}

\section{Ethics approval and consent to participate}

Ethical clearance and approval were obtained ethical and scientific committee of the Asmara College of health Sciences as well as from the Health Research Proposal Review and an Ethical Committee of the Ministry of Health with a reference number of 11/10/17. The members of the ethics committee are namely, Dr. Brhane Debru, Mr. Salih Gemam and Mr. Mehari Weldu. Permission was also secured from both hospitals. After explaining the purpose of the study and assurance of confidentiality and anonymity, informed written consent was obtained from each participant and the interview was conducted. Name and other identifying information were not included in the study.

\section{Consent for publication}

Not applicable

\section{Availability of data and materials}

The datasets generated and/or analyzed during the current study are available from the corresponding author on a reasonable request.

\section{Competing interests}

The authors declare that they have no competing interests. 


\section{Funding}

The research was funded by the National Commission for Higher Education (NCHE). The funder had no role in the design of the project and collection, analysis, and interpretation of data or in writing and publication of the manuscript.

\section{Authors' contributions}

YM: Study conception and design of study, acquisition of data, analysis and interpretation of data, drafting and submission of manuscript; GG: participated in designing and coordination of the study, revising the manuscript critically for important intellectual content; TE: participated in designing and coordination of the study, drafting and revising the manuscript critically for important intellectual content, $\mathrm{EH}$ : design of study, analysis and interpretation of the data, drafting and critical commenting of manuscript. All authors have read and approved the manuscript.

\section{Acknowledgements}

The authors would like to thank the National Commission for Higher Education (NCHE) for funding the research and also the supervisors, data collectors, study participants as well as family and friends for their invaluable support.

\section{Author's information}

${ }^{1}$ Department of Anesthesia and Critical Care, School of Nursing, Asmara College of Health Sciences, Asmara, Eritrea. ${ }^{2}$ Asmara College of Health Sciences, Asmara, Eritrea. ${ }^{3}$ Department of Epidemiology and Biostatistics, School of Public Health, Asmara College of Health Sciences, Asmara, Eritrea.

\section{References}

1. Malczyk D, Marczak K, Miśtal A, Solga K, Borodzicz AM, Krzemińska SA. Are there any unwanted anesthesia symptoms in patients undergoing anesthesia? Journal of Education, Health and Sport. 2018;8(9):77-89.

2. Paavolainen L, Wallstedt J. Post-operative complications of general anesthesia: a recorded video presentation. 2016.

3. Darlene Falco D, Rutledge CDN. Patient Satisfaction With Anesthesia Care: What Do We Know? AANA Journal. 2017;85(4):287. 
4. Abdalrahim MS. Postoperative Pain Assessment and Management: The Effects of an Educational program on Jordanian nurses' practice, knowledge, and attitudes: Institute of Health and Care Sciences; 2009.

5. Fletcher D, Fermanian C, Mardaye A, Aegerter P. A patient-based national survey on postoperative pain management in France reveals significant achievements and persistent challenges. PAIN®. 2008;137(2):441-51.

6. Dumolard P, Gök M, Le N. Nurses' responsibilities in postoperative pain management following total hip arthroplasty. 2017.

7. Savoia G, Alampi D, Amantea B, Ambrosio F, Arcioni R, Berti M, et al. Postoperative pain treatment SIAARTI Recommendations 2010. Short version. Minerva anestesiologica. 2010;76(8):657-67.

8. Joelsson M, Olsson LE, Jakobsson E. Patients' experience of pain and pain relief following hip replacement surgery. Journal of clinical nursing. 2010;19(19-20):2832-8.

9. Frenette L. The acute pain service. Critical care clinics. 1999;15(1):143-50.

10. Chou R, Gordon DB, de Leon-Casasola OA, Rosenberg JM, Bickler S, Brennan T, et al. Management of Postoperative Pain: a clinical practice guideline from the American pain society, the American Society of Regional Anesthesia and Pain Medicine, and the American Society of Anesthesiologists' committee on regional anesthesia, executive committee, and administrative council. The Journal of Pain. 2016;17(2):131-57.

11. Garimella V, Cellini C. Postoperative pain control. Clinics in colon and rectal surgery. 2013;26(03):1916.

12. Stomberg MW, Lorentzen $\mathrm{P}$, Joelsson $\mathrm{H}$, Lindquist $\mathrm{H}$, Haljamäe $\mathrm{H}$. Postoperative pain management on surgical wards-Impact of database documentation of anesthesia organized services. Pain Management Nursing. 2003;4(4):155-64.

13. Gan TJ, Sloan F, de L Dear G, El-Moalem HE, Lubarsky DA. How much are patients willing to pay to avoid postoperative nausea and vomiting? Anesthesia \& Analgesia. 2001;92(2):393-400.

14. Belayneh T, Gebeyehu A, Abdissa Z. Post-operative hypothermia in surgical patients at University of Gondar Hospital, Ethiopia. J Anesth Clin Res. 2014;5(461):2.

15. Stadler M, Bardiau F, Seidel L, Albert A, Boogaerts JG. Difference in risk factors for postoperative nausea and vomiting. Anesthesiology: The Journal of the American Society of Anesthesiologists. 2003;98(1):46-52.

16. Sessler DI. Temperature monitoring and perioperative thermoregulation. Anesthesiology. 2008;109(2):318.

17. Anaesthetists RCo. Guidelines for the provision of anaesthetic services: Royal College of Anaesthetists; 1999.

18. Andemeskel YM, Elsholz T, Gebreyohannes G, Tesfamariam EH. Patient satisfaction with perioperative anesthesia care and associated factors at two National Referral Hospitals: a cross sectional study in Eritrea. BMC health services research. 2019;19(1):669. 
19. Aitkenhead A. Injuries associated with anaesthesia. A global perspective. British journal of anaesthesia. 2005;95(1):95-109.

20. Naidu S, Gopalan P. The informed consent process for anaesthesia: perspectives of elective surgical patients at Inkosi Albert Luthuli Central Hospital, Durban, South Africa. Southern African Journal of Anaesthesia and Analgesia. 2016;22(2):60-4.

21. Jorm C. Patient safety and quality: can anaesthetists play a greater role? Anaesthesia. 2003;58(9):833-4.

22. Barnett SF, Alagar RK, Grocott MP, Giannaris S, Dick JR, Moonesinghe SR. Patient-Satisfaction Measures in AnesthesiaQualitative Systematic Review. Anesthesiology: The Journal of the American Society of Anesthesiologists. 2013;119(2):452-78.

23. Caljouw M, Van Beuzekom M, Boer F. Patient's satisfaction with perioperative care: development, validation, and application of a questionnaire. British journal of anaesthesia. 2008;100(5):637-44.

24. Jlala HA, Caljouw MA, Bedforth NM, Hardman JG. Patient satisfaction with perioperative care among patients having orthopedic surgery in a university hospital. Local and regional anesthesia. 2010;3:49.

25. Tuchscherer J, McKay WP, Twagirumugabe T. Low-dose subcutaneous ketamine for postoperative pain management in Rwanda: a dose-finding study. Canadian Journal of Anesthesia/Journal canadien d'anesthésie. 2017;64(9):928-34.

26. Brennan TJ, Kehlet $H$. Preventive analgesia to reduce wound hyperalgesia and persistent postsurgical pain: not an easy path. Anesthesiology. 2005;103(4):681.

27. Size M, Soyannwo O, Justins D. Pain management in developing countries. Anaesthesia. 2007;62:3843.

28. Apfelbaum JL, Chen C, Mehta SS, Gan TJ. Postoperative pain experience: results from a national survey suggest postoperative pain continues to be undermanaged. Anesthesia \& Analgesia. 2003;97(2):534-40.

29. Dolin S, Cashman J, Bland J. Effectiveness of acute postoperative pain management: I. Evidence from published data. British journal of anaesthesia. 2002;89(3):409-23.

30. El-Nasser GA, Mohamed N. Patient Satisfaction with Preoperative Care and Its Relationship with Patient Characteristics. The Medical Journal of Cairo University. 2013;81(2).

31. Ingabire L. Patients satisfaction with perioperative care at Oshen Fing Feisal Hospital: University of Rwanda; 2017.

32. Kalkman C, Visser K, Moen J, Bonsel G, Grobbee D, Moons K. Preoperative prediction of severe postoperative pain. Pain. 2003;105(3):415-23.

33. Shaikh SI, Nagarekha D, Hegade G, Marutheesh M. Postoperative nausea and vomiting: A simple yet complex problem. Anesthesia, essays and researches. 2016;10(3):388.

34. Lee A, Lum M. Measuring anaesthetic outcomes. Anaesthesia and intensive care. 1996;24(6):685-93.

35. Yentis S, Hartle A, Barker I, Barker P, Bogod D, Clutton-Brock T, et al. AAGBI: Consent for anaesthesia 2017: Association of anaesthetists of great Britain and Ireland. Anaesthesia. 2017;72(1):93-105. 
36. Vyhunthan G, de Silva N. Audit to evaluate preoperative visit to patient by anaesthetist. Sri Lankan Journal of Anaesthesiology. 2012;20(2).

\section{Figures}

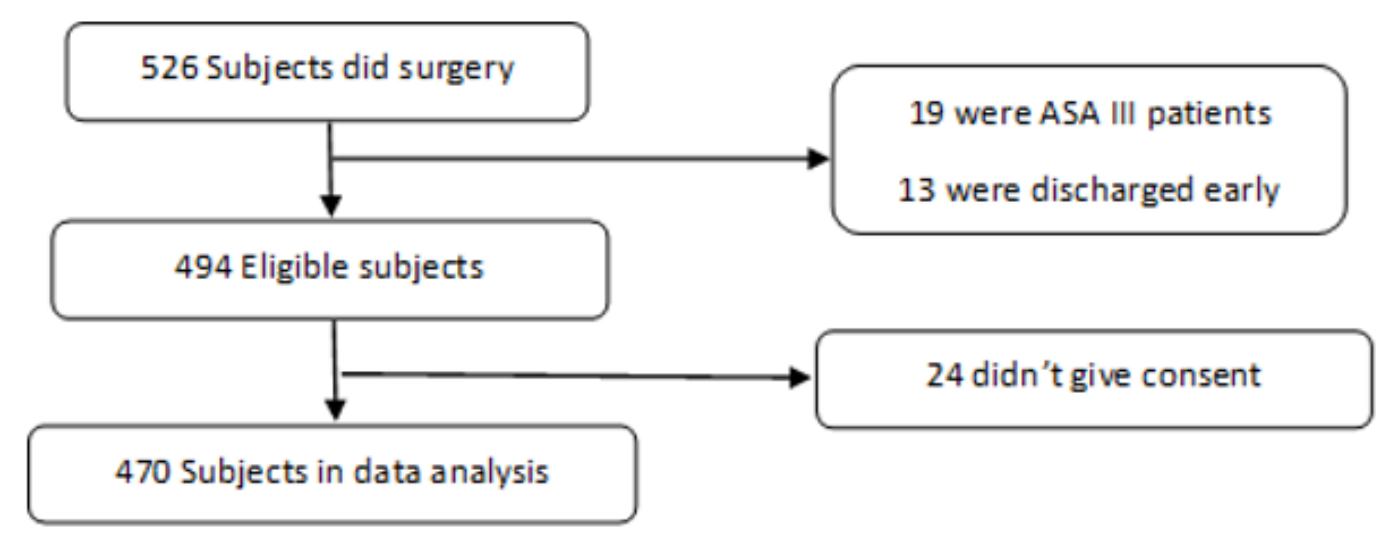

\section{Figure 1}

Number of patients eligible for the study, included in the study, and analyzed 